\title{
La nueva ecología de la información y la documentación en la sociedad del conocimiento: rutinas de elaboración y gestión de contenidos de los diarios digitales españoles ${ }^{1}$
}

\author{
Joaquín LÓPEZ DEL RAMO \\ Universidad Rey Juan Carlos de Madrid \\ joaquin.lopezdelramo@urjc.es \\ María Olivera ZaLdúA \\ Universidad Complutense de Madrid \\ molivera@pdi.ucm.es
}

Recibido: 29/03/2012

Aceptado: 30/10/2012

\begin{abstract}
Resumen
Análisis del tratamiento de los contenidos en los periódicos digitales españoles a través de un cuestionario que evalúa aspectos generales, enlaces, elementos multimedia y recursos participativos. Los medios muestran cierta reticencia a facilitar datos. Se constata un interés prioritario por la introducción de contenidos multimedia en la noticia y como nodos dentro de la cada vez más amplia trama hipertextual. Los contenidos participativos tienen una utilización masiva y creciente, centrada básicamente en las herramientas de microblogging, blogs y comentarios. Las redes más implantadas son Twitter, Facebook, Menéame y Google +. Se considera al lector como eje de los contenidos, hay feed-back regular con él y sus aportaciones se filtran y contrastan. No se observan diferencias claras en estos aspectos entre los periódicos nativos digitales y los inmigrantes.
\end{abstract}

Palabras clave: Periódicos digitales, multimedia, contenidos participativos

\section{The New Ecology of the Information and Documentation in the Knowledge Society: Routines of Elaboration and Management of Contents of the Spanish Digital Newspapers}

\begin{abstract}
We analyze the content of Spanish digital newspapers, using an inquiry form which evaluates general aspects, links, multimedia and social networks. Media appear to be quite reticent about sharing data. One can notice a preference for multimedia contents in news coverage and as junction in the continuously growing hypertextual context. The social networks are more and more massively successful and mainly use tools as microblogging, blogging and commentating. The most popular social netwoks are Twitter, Facebook, Menéame and Google+. They consider the reader as the center of the message. There also is a regular feedback with him and his posts are examined and compared. No clear differences can be observed between digital newspapers from home and abroad.
\end{abstract}

Keywords: Digital newspapers, Multimedia, Social networks

1 Dentro del proyecto de investigación "La nueva ecología de la información y la documentación en la sociedad del conocimiento: desarrollo de una métrica sistémica, planificación de un observatorio para su seguimiento e identificación de tendencias básicas y retos estratégicos (infoscopos.com)". Ministerio de Innovación, Ciencia y tecnología. VI Plan Nacional de I+D+I 2008-2011. Referencia: CSO2009-07619) Dirigido por el profesor Javier García Marco, de la Universidad de Zaragoza. 


\section{Referencia normalizada}

LÓPEZ DEL RAMO, Joaquín y OLIVERA ZALDÚA, María (2013): "La nueva ecología de la información y la documentación en la sociedad del conocimiento: rutinas de elaboración y gestión de contenidos de los diarios digitales españoles”. Estudios sobre el mensaje periodístico. Vol. 19, Núm. 1, págs.: 471-486. Madrid, Servicio de Publicaciones de la Universidad Complutense.

Sumario: 1. Introducción. 2. Objetivos. 3. Metodología. 4. Resultados; 4.1. Aspectos generales sobre la gestión de los contenidos; 4.2. B) Navegación y estructura de la noticia; 4.3. C) Contenidos multimedia; 4.4. D) Contenidos participativos. 5. Conclusiones. 6. Referencias bibliográficas.

\section{Introducción}

Este artículo es fruto de la investigación realizada en el ámbito del proyecto "Infoscopos.com" dentro del módulo sobre medios de comunicación, cuyo equipo está formado por profesores de la Facultad de Ciencias de la Información y Ciencias de la Documentación de la Universidad Complutense de Madrid, y de la Facultad de Ciencias de la Comunicación de la Universidad Rey Juan Carlos. Este grupo está dedicado al exploración de las rutinas de trabajo de los diarios digitales mediante la realización de entrevistas a los responsables de los medios en cuatro grandes ejes temáticos:

1. Elaboración y gestión de contenido

2. Producción

3. Tecnología

4. Archivo y recuperación de la información.

Tras la publicación de los primeros resultados genéricos (Sánchez Vigil et al. 2011a:507-517 y Sánchez Vigil et al. 2011b: 163-712) en este trabajo se aborda como objeto de estudio la primera de las áreas temáticas antes indicada: la elaboración y gestión de los contenidos en los diarios digitales.

Un breve análisis de contexto nos lleva a recordar que la aparición de la prensa digital, mediados los años 90 del pasado siglo, tuvo lugar de forma incierta, como una apuesta a modo experimental o tentativo por parte de algunas empresas periodísticas clásicas que, lógicamente, desconocían el alcance de un medio entonces por completo imprevisible. Los primeros diarios digitales lanzaron un producto que reflejaba la inseguridad con la que nacieron, como una copia simplificada de la edición impresa cuyas páginas se caracterizaban por su parquedad gráfica.

Coincidiendo con las grandes expectativas generadas a partir de la llamada "burbuja digital", de forma relativamente rápida la prensa online va creciendo, y a comienzos de la década 2000 prácticamente todos los diarios importantes estaban ya en la Red. El surgimiento de los periódicos nativos digitales, a pesar de su gran precariedad en medios técnicos y humanos, sirvió de estímulo a las editoras de prensa tradicionales. Progresivamente, en un entorno más competitivo e impulsado por el aumento en la audiencia, el periódico digital empezó a adquirir entidad propia y adopta una configuración con rasgos formales, de arquitectura y de contenido más definidos, aunque aún no del todo perfilados.

La crisis de las empresas.com entre 2001 y 2002 supuso una caída de audiencia y de publicidad, y trajo el fin de algunas iniciativas empresariales en el terreno del pe- 
riodismo digital, así como el cuestionamiento de otras. A partir de 2003 el bache digital empezó a remontarse y, como indica Salaverría (2008: 18-20), en el año 2005 ya había en España más de 1200 medios digitales periodísticos, de los que más de un centenar eran diarios, entre los cuales el $21 \%$ era exclusivos de Internet, y el resto editados por empresas ya existentes de prensa, radio y televisión. Los medios con mayor presencia en Internet eran los impresos, sobre todo los diarios.

A lo largo de todo este periodo, los periódicos digitales siguieron avanzando como producto dotado de particularidades más claramente distintivas respecto al impreso y cada vez más complejas, hasta llegar a un estadio de evolución denominado Periodismo 2.0, en el que el discurso periodístico ya posee rasgos propios y novedosos, basados en cinco atributos esenciales: multimedialidad, hipertextualidad, interactividad, instantaneidad y universalidad (Álvarez Marcos, 2003: 243). Tomando la idea de María Jesús Casals $(2006,64)$ "El camino del conocimiento crea redes, como las ha creado el mundo digital. La posibilidad de los hipervínculos y enlaces ha puesto en evidencia que la comunicación ha dejado su linealidad para ser más parecida a la mente humana: una gran red de relaciones casi infinitas".

El hipertexto y los contenidos multimedia son la base de nuevas modalidades narrativas en el periodismo. Este es un aspecto medular sobre el que incide Canavilhas (2007: 87): "la posibilidad de incorporar nodos de información multimedia en la noticia es una de las marcas más originales del periodismo en la Web [...]. La incorporación de vídeos, sonidos o infografías en la noticia implica cambios en la forma de redactar las noticias y en el diseño de los sitios web". Por su parte, Caminos, Marín y Armentia (Caminos et al. 2007:335) destacan que "los medios digitales no solo interrelacionan textos escritos, sino que, gracias a la versatilidad de las nuevas tecnologías en la Red, han permitido la creación de informaciones en las que se puede integrar texto, sonido, imágenes fijas o en movimiento, infográficos, animaciones en flash, etc., es decir, un texto que es claramente multimedia y que obliga a pensar de qué forma adecuada se pueden estructurar e integrar todas estas potencialidades".

Entre mediados y finales de la pasada década, los diarios online entraron en otro momento expansivo, con audiencias en alza y la aparición de nuevas cabeceras. Se asiste a la consolidación de la prensa nativa de Internet en términos de audiencia, al punto que algunos diarios de este tipo se situaron por encima de muchas cabeceras tradicionales migradas a Internet. Sobre la base de las nuevas capacidades y recursos tecnológicos, se produce una renovación del diseño, tanto en su grafismo como en su arquitectura y modos de navegación, sumado a la incorporación de abundantes contenidos multimedia y nuevos recursos de participación de lector.

Los últimos estudios sobre lectura de diarios en Internet publicados a nivel internacional y en España muestran una tendencia clara y sostenida en el aumento de sus índices de audiencia. Así, según la Asociación de Periódicos Americana (NAA, 212: web), los ciberdiarios de Estados Unidos aumentaron su penetración casi un $6 \%$ en el último cuatrimestre de 2011, alcanzando un récord de 111 millones de visitantes únicos mensuales, 6 millones más que en los mismos meses de 2010. En España, los datos de la OJD Interactiva de enero de 2012 (OJD, 2012: web) son asimismo concluyentes respecto a la elevada lectura de los periódicos digitales, liderados por la edi- 
ción digital de $E l$ Mundo, con más de 30 millones de usuarios únicos mensuales y un promedio diario de casi 2 millones. Además, en general éstos registran niveles de audiencia muy por encima de sus respectivas ediciones impresas.

El retroceso de los diarios de papel frente a los digitales es otra realidad tangible y cada vez más acentuada. En dicho sentido, la reciente investigación "La Prensa: Digital vs Papel 2011" (AIMC, 2011: web), elaborada por la Asociación para la Investigación de los Medios de Comunicación, arroja entre sus principales conclusiones las siguientes:

- Desciende el tiempo que los lectores de diarios impresos dedican a su consulta.

- El lector internauta es un importante consumidor de diarios. El promedio de diarios-papel leídos es de 2,1 títulos diferentes, y 2,9 títulos en prensa online.

- En cuanto a los contenidos, los usuarios prefieren los diarios impresos para leer editoriales, géneros de opinión y temas en profundidad, y el digital para información de actualidad, más rápida y superficial.

Hay una tendencia general a considerar que el contenido juega un papel esencial en el éxito de audiencia de los diarios digitales, y que éste es el verdadero elemento diferenciador que distingue a unos respecto de otros. La tecnología, es común a todos los medios, mientras que el contenido es lo singular. Siguiendo a Casals (ibidem: 64), "el mensaje es el centro. En esta sociedad posindustrial y tecnológica el mensaje es lo esencial y sus fines y efectos serán protagonistas de acciones e investigaciones".

En palabras de Alonso y Martínez (2003b: 274): "la fidelización de los usuarios, que se ha visto potenciada su capacidad de elección y acceso a múltiples fuentes, será determinada, no tanto por las características del medio, sino por los contenidos que resulten más atractivos y sirvan mejor para la satisfacción de sus necesidades". Redunda en la misma idea el trabajo de Rodríguez, Codina y Pedraza (Rodríguez et al., 2010: 36), quienes afirman que "este aumento de consumo de noticias en Internet con la mejora de los contenidos "parece deberse en gran medida al ejercicio de adaptación al formato web que han llevado a cabo los medios de comunicación durante los últimos años. [...] Los sitios web de los medios han aumentado progresivamente la cantidad de contenidos, al tiempo que los han hecho más atractivos".

En la generación de contenidos de los diarios digitales intervienen o cobran fuerza nuevos actores. Una investigación sobre nuevos paradigmas documentales en el periodismo digital (Marcos Recio et al., 2009:61, 62) de cuatro de los principales diarios españoles (El Mundo, El País, Abc y La Vanguardia) indica que "el nuevo paradigma le ofrece al documentalista la posibilidad de participar de forma directa en la creación de contenidos. Para ello ha de aportar elementos informativos y documentales de reciente publicación y que sirvan para complementar la información que propone el periodista".

La eclosión de los contenidos participativos caracteriza la fase en la que nos encontramos hoy, el denominado "Periodismo 3.0", cuya principal virtualidad es que el lector adopta un papel activo como generador del contenido. Todos los estudios recientes coinciden en constatar la idea expresada por Guallar (2011: 103) en el sentido que "los elementos "sociales" (participación y conversación con los lectores), parecen 
ser prioritarios ya para muchos medios, más que los elementos multimedia, cuya incorporación parecería haberse estabilizado o estancado. Así, se ve cómo las herramientas y los productos sociales adquieren más presencia en los rediseños de diarios en 2010, por ejemplo, en los nuevos 20minutos.es o Lavanguardia".

Nadie discute a estas alturas desde las empresas periodísticas la necesidad estratégica de estar en la Web. Sin embargo, desde el punto de vista comercial los diarios digitales no acaban de encontrar una fórmula de viabilidad económica clara. Viven una situación paradójica, en la que el gran aumento del número de lectores y de la publicidad no se traduce aún en ingresos, y por tanto participan de la fortísima crisis económica que atraviesa en general el sector de medios de comunicación.

\section{Objetivos}

Inmersos en el contexto que acaba de describirse, los diarios digitales muestran en la Red su fachada externa, en la que usualmente se centran los análisis de los investigadores, pero no es tan frecuente la indagación de la parte interna, cuyas estrategias y acciones explican lo que de ellos vemos en la esfera pública de Internet. Ese es, precisamente el foco de interés de este trabajo, cuyos principales objetivos son:

1. Determinar la importancia que los diarios digitales otorgan a los contenidos multimedia y saber quiénes son sus responsables.

2. Conocer los criterios empleados por los ciberdiarios respecto al tratamiento estructural la noticia basado en la hipertextualidad.

3. Indagar respecto a la inclusión de los elementos multimedia de superficie que integran la noticia.

4. Examinar cómo se gestionan los contenidos participativos que incluyen los diarios y cuál es su tipología.

5. Comprobar si existen diferencias acusadas en el tratamiento de todos los aspectos anteriores entre los diarios nativos e inmigrantes de Internet.

\section{Metodología}

Para la selección de la muestra representativa se tomaron como punto de partida los periódicos posicionados por volumen de audiencia en los 20 primeros lugares de los dos principales índices españoles: OJD y EGM. Una vez obtenidos, entre los mismos se realizó una selección basada en los siguientes criterios:

- Se eligieron periódicos generalistas, excluyendo los gratuitos, así como deportivos o económicos, por las especificidades que presentas éstos, lo cual podría romper la necesaria unidad y coherencia de la muestra.

- Se analizó un periódico por grupo de comunicación, para evitar redundancias, por las posibles sinergias de trabajo que pudieran darse.

- A los seleccionados en base a los anteriores criterios, se añadió el periódico de más difusión (tomando como referencia la OJD Interactiva) de los grupos Joly y Promecal por la importancia de éstos, aunque en los índices de audiencia están situados en lugares más bajos.

- Un máximo de dos periódicos nativos digitales, que en este caso son los líderes de audiencia. 
Con estos requerimientos, la muestra seleccionada quedó finalmente establecida en 21 periódicos, a saber: Abc, Canarias 7, Diario de Burgos, Diario de Navarra, Diario de Sevilla, El Confidencial, El Día, El Mundo, El País, El Periódico de Cataluña, Heraldo de Aragón, Información, La Nueva España, La Opinión de Coruña, La Razón, La Vanguardia, La Voz de Galicia, Levante, Libertad Digital, Público y Última Hora

Desde el punto de vista instrumental, se procedió a la elaboración de un cuestionario de diseño propio y organizado en cuatro partes, correspondientes a las áreas temáticas consideradas: contenidos, producción, tecnología y documentación, a fin de poder ser respondidos de forma independiente por responsables de dichas áreas. Se eligió este instrumento metodológico por ser el único adecuado a los fines de la investigación, ya que se trata de datos que por lo común no están disponibles públicamente, y era necesario recurrir a fuentes internas para obtener la información.

El cuestionario sobre elaboración y gestión de contenidos, tema concreto que concierte a este trabajo, quedó configurado finalmente por 18 preguntas, a su vez agrupadas en cuatro apartados: Aspectos generales, Navegación y estructura de la noticia, Contenidos multimedia y Contenidos participativos, distribuyéndose de la siguiente forma:

-A. Aspectos generales

- A.1. ¿Qué importancia se da en su medio a los nuevos contenidos en formato digital: (fotografía, vídeo, documentos sonoros, etc.) frente a los tradicionales?: Elevada, Media, Escasa, Nula.

- A.2. ¿Cómo se llama la figura que determina con preferencia los contenidos finales?: Editor, Productor, Otros.

- A.3. Factores que más influyen en la elección del contenido: Lector, Línea editorial, Publicidad, Interés del grupo.

- A.4. ¿Reciben apoyos documentales las secciones que se indican a continuación?: Cultura, Deporte, Nacional, Economía, Internacional

- A.5. Con respecto a la pregunta anterior, indique si las mencionadas secciones reciben distinto grado de apoyo documental y cuantifíquelo.

- B. Navegación y estructura de la noticia

- B.1. ¿Qué pauta se sigue en cuanto a la inclusión de diferentes tipos de enlaces en las informaciones?: Elementos de archivo, Entre secciones, Entre ediciones, Externos, Galerías, Videos, Todos los anteriores

- B.2. ¿Hay interconexión mediante enlaces entre noticias o páginas pertenecientes a diferentes secciones del periódico si aluden a un mismo hecho informativo?. En caso afirmativo, indíquese en qué secciones es más habitual.

- B.3. Indique las nuevas formas de presentar la información empleadas en su periódico: Galerías, Videos, Multimedia, Links, Infografías, Ninguna, Todos.

- B.4. ¿Cómo definiría esta fórmula de presentar los contenidos: Nuevo género, Información tradicional

- B.5. Si es un nuevo género, ¿cómo se llamaría?: Periodismo digital, Periodismo multimedia, A decidir 
- C. Contenidos multimedia

- C.1. ¿Cuáles son los criterios para la introducción de elementos multimedia en la noticia?: Generales, Específicos (Prioridad al texto, Prioridad a la imagen, Mixto, Prioridad al multimedia, otros).

- C.2. ¿Establece limitaciones en cuanto a la inclusión de contenidos gráficos o audiovisuales? En caso afirmativo, cítelas.

- C.3. ¿Qué valor da su medio a la fotografía frente al vídeo?: Mayor, menor o semejante

- D. Contenidos participativos

- D.1. Tipos de herramientas que permiten generar contenidos a los lectores y tienen cabida en el periódico: Blogs, Fotos, Chats, Galería, Microblogging, Otros (Indique)

- D.2. ¿Existe algún tipo de filtrado de este contenido?

- D.3. ¿Se admite la contribución de los lectores para cubrir una noticia?. En caso afirmativo indique los criterios empleados.

- D.4. ¿Hay feed-back (reciprocidad) con el lector y en qué consiste?. En caso afirmativo indicar en qué consisten

- D.5. ¿A qué redes sociales está vinculado el periódico?

En relación al procedimiento, de los 21 periódicos seleccionados respondieron al cuestionario sobre contenidos 12 (57, 14\%): Diario de Navarra, El Confidencial, El Mundo, El País, El Periódico de Cataluña, Heraldo de Aragón, Información, La Nueva España, La Opinión de Coruña, La Voz de Galicia, Levante y Libertad Digital. En ningún caso hubo respuesta negativa expresa a la colaboración sino que, a pesar de la insistencia, simplemente no hubo respuesta.

Las respuestas no fueron inmediatas sino intermitentes, $\mathrm{y}$ a veces se dieron solo a una de las partes de la encuesta, es decir, no respondieron a todos los temas, casos de $A b c$, El Mundo y La Nueva España. El tiempo transcurrido entre el envío de la encuesta y la respuesta fue en general largo, lo que obligó a un constante seguimiento, con reiteradas peticiones a los responsables. Esta situación ralentizó y bloqueó en ocasiones el normal desarrollo de la tarea. El periodo de respuesta por parte de los medios se extendió entre los meses de abril y octubre de 2011.

\section{Resultados}

Se exponen a continuación los resultados obtenidos en cada una de las preguntas; en algunos casos, se agrupan los resultados en un mismo cuadro en aras a una mayor claridad.

\subsection{A) Aspectos generales sobre la gestión de los contenidos:}

- A.1. Qué importancia se da en su medio a los nuevos contenidos en formato digital: (fotografía, vídeo, documentos sonoros, etc.) frente a los tradicionales?:

El 83, 3\% de los periódicos declaran otorgar el máximo valor a los contenidos digitales, y solo dos de ellos, (Heraldo de Aragón y La Nueva España), equivalentes al 16 ' $7 \%$, consideran que la importancia de estos nuevos contenidos tiene un valor mediano frente a los tradicionales. Ninguno declaró un interés bajo o nulo. 
- A.2 y A.3 ¿Cómo se llama la figura que determina los contenidos finales y quién influye más en la elección de los mismos?:

Tienen cabida opciones de respuesta simultánea. La lectura de los datos que se muestran en la Tabla I permite apreciar diferentes aspectos:

1. Si hay un único responsable en la elección del contenido o son varios: en este caso encontramos que en nueve de los periódicos digitales analizados, es decir, un $75 \%$, hay un responsable único; ocho de ellos lo denominan "responsable de contenidos" y uno (El Confidencial) con el tradicional nombre de "editor". En el 25\% (El País, La Opinión de Coruña y Libertad Digital), la responsabilidad es compartida, aunque no del mismo modo, pues en El País es más restringida y en los otros dos es más amplia.

2. Respecto a la elección de contenido, se observa que para el $75 \%$ de los diarios el factor que más influye es el lector. Del resto de casos, solo en uno (La Nueva España) encontramos que lo principal es la línea editorial del medio, y en otros dos $(16,6 \%)$, aunque declaran que el lector sí cuenta, no es el único factor de la influencia, para El Periódico de Cataluña también incide la línea editorial, mientras que Libertad Digital es el medio mas abierto a este respecto, al declarar que todos los factores considerados como respuesta posible (lector, línea editorial, publicidad e interés del grupo) tienen incidencia en lo que afecta a esta cuestión.

Tabla I: Responsabilidad e influencia en la elección de contenidos. Elaboración propia

\begin{tabular}{|l|l|l|l|l|l|l|l|}
\hline Diarios & $\begin{array}{l}\text { Responsable } \\
\text { de contenido }\end{array}$ & Editor & Todos & Lector & $\begin{array}{l}\text { Línea } \\
\text { editorial }\end{array}$ & Publicidad & $\begin{array}{l}\text { Interés del } \\
\text { grupo }\end{array}$ \\
\hline Diario de Navarra & & & & & & & \\
\hline El Confidencial & & & & & & & \\
\hline El Mundo & & & & & & & \\
\hline El País & & & & & & & \\
\hline El Periódico de Catalunya & & & & & & & \\
\hline Heraldo de Aragón & & & & & & & \\
\hline Información & & & & & & & \\
\hline La Nueva España & & & & & & & \\
\hline La Opinión de A Coruña & & & & & & \\
\hline La Voz de Galicia & & & & & & & \\
\hline Levante & & & & & & \\
\hline Libertad Digital & & & & & & & \\
\hline
\end{tabular}

- A.4 y A.5 ¿Qué secciones reciben apoyos documentales?, y ¿cuál es el porcentaje del apoyo en cada caso?

Para la primera de estas cuestiones, las respuestas posibles eran asimismo de múltiple elección, y se centran en las secciones que normalmente tienen cabida en todos los medios periodísticos de información general: Cultura, Deporte, Nacional, Economía e Internacional, etiquetas que representan los principales campos temáticos informativos. No contestan a esta pregunta los responsables de El País y La Voz de Galicia. Los resultados reflejan que todas ellas reciben apoyo documental, pero matizando por secciones y medios concretos se observan los siguientes datos:

1. No hay grandes diferencias entre las distintas secciones. La que cuenta con refuerzo documental en más periódicos es Deportes, sumando un $90 \%$ de los casos, seguida por Cultura, Nacional, Economía e Internacional, a las que corresponde un mismo valor porcentual del $70 \%$. 
2. Por medios, dan apoyo documental a todas las secciones El Mundo, El Periódico de Cataluña, Información, La Nueva España, y Libertad Digital. Los periódicos que refuerzan documentalmente algunas secciones son Diario de Navarra, El Confidencial, Heraldo de Aragón, La Opinión de Coruña y Levante y lo hacen de forma desigual: tres en la sección Deporte, y dos respectivamente en Cultura, Nacional, Economía e Internacional. El diario digital que ofrece este apoyo a menos secciones es $\mathrm{He}$ raldo de Aragón, únicamente a Deportes.

Una vez conocidas las secciones que cuentan con soporte documental, interesa descubrir en qué grado se produce éste. Tomando como referencia el conjunto de la muestra es Deportes la que sale más beneficiada, pues supera a las otras en número de periódicos (Diario de Navarra, El Periódico de Cataluña, Heraldo de Aragón y La Opinión de Coruña) y además le corresponde la media total más alta de apoyo, cifrada en el 35\%. La segunda sección en orden de resultados es Economía, la más reforzada documentalmente en los diarios El Confidencial y La Opinión de Coruña (en este al mismo nivel que Deportes), y tiene un porcentaje medio del 27'5\%. El tercer lugar se sitúa la sección Nacional, cuyo mayor apoyo está en Levante y tiene un peso global del $26 \%$. Viene a continuación Internacional, con una media del $24,1 \%$ y solo es la sección más reforzada en El Mundo. Cultura es la que recibe un menor aporte documental, situado en una media del $16,6 \%$, y no lidera el ranking en ningún diario.

Por cabeceras, solo dos prestan el mismo apoyo a todas las secciones: Información y Libertad Digital. En El Periódico de Cataluña y Levante las diferencias entre unas secciones y otras no son muy acusadas, mientras que en el resto hay notables desigualdades.

\subsection{B) Navegación y estructura de la noticia}

- B.1 ¿Qué pauta se sigue en cuanto a la inclusión de diferentes los tipos de enlaces en las informaciones?

Se plantean como posibles tipos de enlaces los siguientes: Elementos de archivo, Vínculos entre secciones, Vínculos entre ediciones distintas del periódico, Enlaces externos, Galerías, y Vídeos. Las respuestas se refieren a la praxis o norma habitual, con la salvedad de que pueden existir acontecimientos o noticias concretas en los que los periódicos procedan de otra manera, es decir, se trata de pautas en parte susceptibles de modificación.

Lo relevante en este caso era conocer si los periódicos tienden como estrategia dotar a las informaciones de mayor o menor riqueza hipertextual. Los resultados obtenidos son contundentes ya que un $83,3 \%$ de los periódicos emplean todos los tipos de enlaces considerados, y el 16,6\% restante prescinden solo de los enlaces a sitios externos.

- B.2 Interconexión de enlaces entre noticias o páginas de diferentes secciones si aluden al mismo hecho informativo. En caso afirmativo, indíquese en qué secciones es una práctica más habitual.

Un $75 \%$ de los periódicos contestaron afirmativamente: Diario de Navarra, El Confidencial, El Periódico de Cataluña, Heraldo de Aragón, Información, La Nueva España, La Opinión de Coruña, La Voz de Galicia y Levante, mientras que respon- 
dieron de forma negativa el $16{ }^{\prime} 5 \%$, El Mundo y Libertad Digital, y no se manifestó El País.

La sección donde con más frecuencia se da esta práctica es Nacional ( $70 \%$ de los diarios que contestaron a la pregunta), seguida de Deportes (50\%), Economía (30\%), Internacional (20\%) y Cultura (1\%).

- B.3 Nuevos elementos empleados para presentar la información

Esta pregunta alude directamente a una de las características definitorias del periodismo digital: la multimedialidad. La observación de los resultados en la Tabla II arroja como idea general que el empleo de estos elementos está ampliamente generalizado y diversificado. Todos los medios analizados los utilizan y lo hacen casi en todas sus variantes. Hay unanimidad en cuanto a la utilización de galerías fotográficas, vídeos y links. Salvo Levante, los diarios incluyen también elementos de naturaleza estrictamente multimedia, mientras que el recurso que registra un menor índice de empleo es la infografía, ausente en el $25 \%$ de los casos.

Por diarios, se aprecia que ocho de los 12 que facilitaron datos, equivalentes al $66,6 \%$, utilizan la totalidad de los nuevos elementos en la confección de sus informaciones. El Confidencial, El Periódico de Cataluña y Heraldo de Aragón coinciden en prescindir de las infografías, aunque sí hacen uso de los demás.

Tabla II: Nuevos elementos para presentar la información. Elaboración propia

\begin{tabular}{|l|l|l|l|l|l|}
\hline \multicolumn{1}{|c|}{ Diarios } & Galerías & Videos & Multimedia & Links & Infografías \\
\hline Diario de Navarra & & & & & \\
\hline El Confidencial & & & & & \\
\hline El Mundo & & & & & \\
\hline El País & & & & & \\
\hline El Periódico de Catalunya & & & & & \\
\hline Heraldo de Aragón & & & & & \\
\hline Información & & & & & \\
\hline La Nueva España & & & & & \\
\hline La Opinión de A Coruña & & & & & \\
\hline La Voz de Galicia & & & & & \\
\hline Levante & & & & & \\
\hline Libertad Digital & & & & & \\
\hline
\end{tabular}

- B.4 y B.5 Consideración y definición de los nuevos contenidos

Dos preguntas incluidas en el cuestionario a fin de tener una referencia conceptual de los propios medios sobre los nuevos contenidos del periodismo digital. La primera cuestión, si consideran que la utilización de estos elementos como parte de la narrativa informativa propia de los ciberdiarios supone en realidad el surgimiento de un nuevo género, recibe una respuesta afirmativa del $75 \%$ de los periódicos: Diario de Navarra, El Confidencial, El Mundo, Heraldo de Aragón, Información, La Nueva España, La Opinión de Coruña, La Voz de Galicia y Libertad Digital. Solo El Periódico de Cataluña y Levante, representativos del 16'6\%, entienden que no se trata de un nuevo género, mientras que El País no contesta.

Mayor dispersión se observa en cuanto al término o términos para definir este nuevo género, pregunta que se formulaba con respuesta abierta. El 41,6\% de los periódicos (El Mundo, Heraldo de Aragón, La Opinión de Coruña, Levante y Libertad 
Digital) consideran que la definición más adecuada es "Periodismo multimedia". Para el 33,5\% de los medios encuestados (Diario de Navarra, El Confidencial, Información de Alicante y La Nueva España) lo más preciso es hablar de "Periodismo digital". Solo La Voz de Galicia respondía a esta pregunta con una definición propia: "Periodismo multiplataforma".

\subsection{C) Contenidos multimedia}

- C.1 ¿Cuáles son los criterios para la introducción de elementos multimedia en la noticia?

En la Tabla III se visualizan los datos correspondientes, apreciándose de forma nítida que la mayoría de medios ( $72,7 \%$ de los que responden) utilizan criterios generales. La diferencia respecto al resto es notable, pues se prioriza el propio elemento multimedia únicamente en cuatro casos $(36,5 \%)$, tres de los periódicos dan prevalencia a la imagen $(27,2 \%)$ y solo uno adopta una solución mixta. Ninguno de ellos elige el texto como elemento prioritario ni otras fórmulas diferentes.

Tabla III: Criterios para la introducción de elementos multimedia en la noticia. Elaboración propia

\begin{tabular}{|l|l|l|l|l|l|}
\hline \multicolumn{1}{|c|}{ Diarios } & Generales & $\begin{array}{c}\text { Prioridad } \\
\text { al texto }\end{array}$ & $\begin{array}{c}\text { Prioridad a } \\
\text { la imagen }\end{array}$ & Mixto & $\begin{array}{c}\text { Prioridad al } \\
\text { multimedia }\end{array}$ \\
\hline Diario de Navarra & & & & & \\
\hline El Confidencial & & & & & \\
\hline El Mundo & & & & & \\
\hline El País & & & & & \\
\hline El Periódico de Catalunya & & & & & \\
\hline Heraldo de Aragón & & & & & \\
\hline Información & & & & & \\
\hline La Nueva España & & & & & \\
\hline La Opinión de A Coruña & & & & & \\
\hline La Voz de Galicia & & & & & \\
\hline Levante & & & & & \\
\hline Libertad Digital & & & & & \\
\hline
\end{tabular}

- C.2 ¿Establece limitaciones en cuanto a la inclusión de contenidos gráficos o audiovisuales? En caso afirmativo, citarlas.

Siete periódicos responden que no existen estas limitaciones, concretamente Diario de Navarra, El Confidencial, El País, El Periódico de Cataluña, La Nuevas España, La Voz de Galicia y Libertad Digital, que suponen el 58,3\% del total. Los que contestan en sentido contrario suman el 41,6\% Dentro de este grupo, no todos citan expresamente cuáles son las limitaciones; entre los que sí lo hacen, el más concreto en la respuesta es El Mundo, que se centra en el exceso de peso total de los archivos, marcando un número no mayor de 15-20 videos, no más de 10-12 galerías y no más de seis fotos por galería, o la proporción conjunta equivalente. El Heraldo de Aragón solo especifica que impone limitaciones técnicas. Información pone como barrera la saturación o repetición de contenidos. En La Opinión de Coruña "el peso total de la página no puede exceder los límites establecidos", pero no señala cuáles son éstos. Por último, para Levante el único límite es la exclusión de infografías. 
- C.3 ¿Qué valor da su medio a la fotografía frente al vídeo?

La respuesta se acota a tres opciones: Mayor, Menor o Semejante. El 66,6\%, dan más importancia a la fotografía que el vídeo. Ocurre con contrario solo en dos casos (16,6\%), que corresponden a El Mundo e Información. Mientras, El Periódico de Cataluña y La Nueva España, responden que dan el mismo valor a la fotografía y al vídeo.

\subsection{D) Contenidos participativos}

- D.1 Tipos de herramientas que permiten generar contenidos a los lectores y tienen cabida en el periódico.

La lectura general de los datos de la Tabla IV nos indica dos aspectos destacados: el empleo de estas utilidades en los diarios digitales está muy extendido y se usan todas las herramientas disponibles, aunque con diferente proporción. Destacan en las primeras posiciones, por este orden: Microbolgging, Comentarios, Blogs y Fotografías.

Las herramientas más empleadas son el microblogging y los comentarios, disponibles en los 12 periódicos examinados. Le siguen los blogs, que tienen cabida en 10 diarios ( $83,3 \%$ del total). A continuación se encuentra la inserción de fotografías, que existe en el $75 \%$ de los periódicos. En un escalón inferior están los chats, disponibles en cinco medios $(41,6 \%)$ y las galerías de lectores $(33,3 \%)$. El tipo de herramientas participativas menos representativo lo integran lo foros de debate y las noticias de lectores, que se dan en el $16,6 \%$ de los casos.

Por periódicos, encontramos que en cuatro de ellos (Heraldo de Aragón, La Nueva España, Levante y Libertad Digital), equivalentes al 33,3\%, se incluyen todos los tipos de contenidos participativos principales. El País solo excluye las galerías y los comentarios. Por su parte, Diario de Navarra y La Voz de Galicia prescinden de chats y galerías, cosa que también hace Información, que es uno de los que tampoco dan cabida a los comentarios. La Opinión de Coruña y El Mundo poseen blogs, a los que suman también comentarios y foros. Por último, el periódico que menos herramientas de participación utiliza es el nativo El Confidencial, que dispone únicamente del microblogging y comentarios.

Tabla IV: Herramientas de generación de contenidos por parte de los lectores. Elaboración propia

\begin{tabular}{|l|l|l|l|l|l|l|}
\hline \multicolumn{1}{|c|}{ Diarios } & Blogs & Fotos & Chats & Galería & Microblogging & \multicolumn{1}{c|}{ Otros } \\
\hline ABC & & & & & & Comentarios \\
\hline Diario de Navarra & & & & & & Comentarios \\
\hline El Confidencial & & & & & & $\begin{array}{l}\text { Comentarios } \\
\text { Encuestas }\end{array}$ \\
\hline El Mundo & & & & & & Comentarios \\
\hline El País & & & & & & $\begin{array}{l}\text { Comentarios } \\
\text { Encuestas }\end{array}$ \\
\hline El Periódico de Catalunya & & & & & & Comentarios \\
\hline Heraldo de Aragón & & & & & & Comentarios \\
\hline Información & & & & & & Comentarios \\
\hline La Nueva España & & & & & & Comentarios, Foros \\
\hline La Opinión de A Coruña & & & & & & Noticias, Comentarios Debates, Concurso \\
\hline La Voz de Galicia & & & & & & Comentarios \\
\hline Levante & & & & & & Comentarios Noticias \\
\hline Libertad Digital & & & & & &
\end{tabular}


- D.2 ¿Existe algún tipo de filtrado de este contenido?

Todos los periódicos consultados contestan afirmativamente a esta pregunta

- D. 3 ¿Se admite la contribución de los lectores para cubrir una noticia?. En caso afirmativo indicar con qué criterios.

De los 12 medios que cumplimentaron la encuesta solo El País dejó en blanco esta pregunta. La respuesta mayoritaria fue afirmativa en ocho de los 11 periódicos restantes, lo que supone el 72,7\%, y los otros tres (El Mundo, El Periódico de Cataluña y Levante) contestaron negativamente.

Respecto a los criterios que emplean aquellos medios que admiten contribuciones de los lectores, las respuestas fueron muy variadas. Diario de Navarra no indicó nada sobre el particular. El Confidencial centró su respuesta en el canal empleado, señalando que la aportación del lector se realiza a través de los comentarios. Heraldo de Aragón valora la calidad de lo aportado y la veracidad. Información de Alicante admite como fuente al lector pero condiciona su posible aportación al contenido a su contraste por parte del periodista. La Voz de Galicia sigue el mismo procedimiento anterior: el lector propone un tema, envía un texto o sube una foto, pero antes de publicar el contenido siempre es revisado por el periodista. La Nueva España afirma que cualquier dato que faciliten los lectores es tenido en cuenta, pero no explica criterios de filtrado; de forma muy similar se pronuncia La Opinión de Coruña. Por último, Libertad Digital restringe estas contribuciones de lector a casos muy excepcionales.

- D.4 ¿Hay feed-back (reciprocidad) con el lector y en qué consiste?

Como en la cuestión anterior, El País no contestó. Todos los demás medios responden de manera afirmativa. En relación a la segunda parte de la pregunta, Diario de Navarra no aporta ningún comentario, y los demás medios indican lo siguiente:

- El Confidencial: "Muy frecuentemente el periodista y el lector contactan a través de los comentarios, correo, facebook y twitter".

- Heraldo de Aragón: "Comentarios a la noticia, y nosotros podemos observar si interesa o no través de las más vistas".

- El Mundo: "Continuo diálogo sobre sus demandas y sugerencias, abierto o por correo".

- El Periódico de Cataluña: "Pedir opinión o reacciones sobre determinados hechos"

- Información de Alicante: "Damos vías de contacto fácil y continuado con el lector"

- La Opinión de Coruña: "Nos comunicamos con los lectores a través de las redes sociales y de los comentarios de las noticias".

- La Voz de Galicia: "Opiniones sobre las noticias, noticias enviadas por los lectores, concursos"

- Levante: "Comentarios y extensiones a redacción si hace falta un profesional".

- Libertad digital: "En las redes sociales".

-D.5 ¿A qué redes sociales está vinculado el periódico?

En las respuestas recibidas únicamente se indica si el periódico enlaza con las redes más extendidas (Facebbok, Twiter, Tuenti, Youtube, Menéame, Delicious, Myspace, 
Digg, Eskup y Google +) pero no se especifica un matiz importante: si el medio cuenta con perfil propio en la red (no todas tienen esa propiedad o configuración) o si simplemente se enlaza con ella para que el lector comparta las noticias. A fin de obtener unas conclusiones más exactas, los autores han procedido a constatar empíricamente este extremo, lo que se refleja asimismo en los resultados que se exponen a continuación.

El dato general más relevante es que todos los periódicos poseen vínculos con algún tipo de red social. Teniendo en cuenta las 10 que se han considerado en el cuestionario, los ciberdiarios que enlazan con mayor número de redes lo hacen con seis, El Mundo, El País, El Periódico de Cataluña, La Voz de Galicia y Levante. Con vínculo a cinco de las mencionadas redes se encuentran Diario de Navarra, Heraldo de Aragón, Información de Alicante y Libertad Digital. El último lugar de esta escala lo ocupan El Confidencial, La Nueva España y La Opinión de Coruña, que presentan enlace con cuatro redes. El cómputo global arroja una media ligeramente superior a cinco redes por periódico, siempre en referencia a las consideradas en el estudio, pues hay medios vinculados a otras redes de menor implantación o difusión.

Desde el punto de vista de las redes, una de las que cuenta con mayor implantación es Twitter, donde todos los periódicos de la muestra están vinculados y cuentan con perfil propio. Le sigue Facebook, también con el 100\% de medios enlazados, si bien uno de ellos, Información de Alicante, no posee perfil propio. Otra de las redes a las que enlazan todos los periódicos es Menéname, cuya función específica es compartir noticias. También cuenta con una gran implantación Google + , herramienta para recomendar noticias, que está presente en el 91,6\% de los medios analizados. Le sigue en orden descendente Tuenti, a la que se vincula el 58,3\% de los cibermedios consultados. En una escala mucho más minoritaria se sitúan Youtube (25\%), Delicious $(16,6 \%)$ y, empatadas al 8,3\%, Myspace y Eskup.

Respecto a otras redes sobre las que no se preguntó, destaca Linkedin por estar enlazada al $16,6 \%$ de los periódicos. Se registra la presencia puntual de Fresqui, LiveSpace y Flickr.

\section{Conclusiones}

1. Los responsables de los diarios digitales se muestran algo reacios a facilitar información sobre las rutinas de trabajo y políticas de gestión de contenido. Una parte significativa no responde o lo hace parcialmente, y en otros casos se requiere mucha insistencia para obtener respuesta.

2. Los diarios digitales otorgan a los nuevos contenidos multimedia una elevada importancia frente a los tradicionales. El lector es el factor que más influye para ellos en la elección de estos contenidos, por encima de la línea editorial, los intereses del grupo o la publicidad.

3. La documentación es un importante factor de apoyo o refuerzo en los contenidos de los diarios digitales, con una incidencia notable en las principales secciones informativas. El aporte documental es especialmente significativo en deportes, seguida de economía e información nacional.

4. Domina la tendencia de incorporar en las informaciones enlaces a elementos muy variados. De ello se desprende que se amplían los recorridos y tramas de nave- 
gación por un mismo tema o noticia a través de nuevos elementos, siendo éstos de diferente naturaleza, ya no solo textual sino también, y en gran media, gráficos.

5. Se constata la preferencia por el empleo generalizado de elementos multimedia como componente de las informaciones, en toda su variedad de posibilidades.

6. Una mayoría muy significativa de los medios digitales consideran que la integración de nodos y contenidos multimedia como elementos esenciales de la narrativa periodística supone en realidad en nacimiento de un nuevo género, al que optan por denominar, aproximadamente al 50 por ciento, "Periodismo Multimedia" o "Periodismo Digital".

7. No hay un criterio muy definido para la introducción de elementos multimedia, y solo en casos concretos se da prioridad al propio elemento por encima de otras consideraciones. Gran parte de los medios consultados indican que no tienen limitaciones establecidas a la hora de incorporarlos, mientras que en otros casos establecen una restricción de capacidad física, determinada por el número y el peso de los archivos. En general, se da más valor a la fotografía que al vídeo.

8. Los contenidos participativos están implantados en el 100\% los periódicos. Las herramientas más empleadas son: microblogging, comentarios de noticias, blogs, y fotos de lectores. Los servicios de microblogging han desplazado a los blogs y los chats como recurso mayoritario de participación.

9. Todos los diarios filtran los contenidos generados por los lectores. Un porcentaje significativo incluso dice admitir las contribuciones de estos para cubrir noticias, aunque sujetas a contraste por parte de los periodistas del medio. En todos los casos, hay feed-back con el lector, especialmente a través del correo electrónico, comentarios y redes sociales. Las redes más implantadas son Twitter, Facebook, Menéame y Google + , aunque se van incorporando con rapidez otras nuevas.

10. No se han apreciado particulares diferencias en las rutinas de tratamiento del contenido en los diarios nativos digitales y los inmigrantes del ámbito impreso.

\section{Referencias bibliográficas}

AIMC (Asociación para la Investigación de los Medios de Comunicación, 2011): $L a$ Prensa: Digital vs. Papel. En: http://www.aimc.es/-La-Prensa-Digital-vs-Papel.html [fecha de consulta: 20 de febrero de 2012]

ALONSO, Jaime y MARTÍNEZ, Lourdes (2003): "Medios interactivos: caracterización y contenidos", en DÍAZ NOCI, Javier y SALAVERRÍA, Ramón (eds.): $M a$ nual de Redacción Ciberperiodística. Barcelona, Ariel, pp. 261-305.

ÁLVAREZ MARCOS, José (2003): "El Periodismo ante la tecnología hipertextual", en DÍAZ NOCI, Javier y SALAVERRÍA, Ramón (eds.): Manual de Redacción Ciberperiodística. Barcelona, Ariel Comunicación, pp. 231-258

CAMINOS, José Ma; MARIN, Flora y ARMENTIA, José Ignacio (2007): "Elementos definitorios del periodismo digital". Estudios sobre el mensaje periodístico, vol. 13. Madrid, Servicio de Publicaciones de la Universidad Complutense, pp. 316-336. 
CANAVILHAS, Joao (2007): Webnoticia: Propuesta de modelo periodístico para la $w w w$. Covilhá, Universidade da Beira Interior, Libros Labcom.

CASALS CARRO, María Jesús (2006): "La enseñanza del periodismo y las nuevas tecnologías de la información y la comunicación". Estudios sobre el mensaje periodístico, vol. 12, Madrid, Servicio de Publicaciones de la Universidad Complutense, pp. 59-70.

GUALLAR, Jorge (2011): "Prensa digital en 2010". Anuario ThinkEPI, vol. 5, pp. 101-105.

MARCOS RECIO, Juan Carlos; SÁNCHEZ VIGIL, Juan Miguel y SERRADA, María (2009): "Nuevos paradigmas periodísticos y documentales en los periódicos digitales: estudio de casos en España". Investigación Bibliotecologica, vol. 23, $\mathrm{n}^{\circ}$ 49. México, pp. 43-65.

NNA (Newspaper Association of America): Febrero 2012: http://www.naa.org/Newsand-Media/Press-Center/Archives/2012/Growth-Tren Tabla IV: Herramientas de generación de contenidos por parte de los lectores. Fuente: elaboración propia dContinues-For-Newspaper-Websites.aspx. [fecha de consulta: 11 de febrero de 2012]

OJD Interactiva (2012): http://www.ojdinteractiva.es/ultimos-medios-auditados.php\# (Enero) [fecha de consulta: 25 de febrero de 2012]

RODRÍGUEZ-MARTÍNEZ, Ruth; CODINA, Lluis y PEDRAZA-JIMÉNEZ, Rafael (2010): "Cibermedios y web 2.0: modelo de análisis y resultados de aplicación". El Profesional de la Información, 2010, enero-febrero, v.19, n 1, pp. 35-44.

SALAVERRÍA, Ramón (2008): "La investigación sobre ciberperiodismo en España: tendencias, resultados y perspectivas", en LÓPEZ GARCÍA, Guillermo (ed.): Comunicación local y nuevos formatos periodísticos en Internet: cibermedios, confidenciales y weblogs. Valencia, Servei de Publicacions de la Universitat de Valencia, pp. 15-34. Disponible en http://www.cibermediosvalencianos.es/comloc/Salaverria.pdf [fecha de consulta: 5 de febrero de 2012].

SÁNCHEZ VIGIL, Juan Miguel; MARCOS RECIO, Juan Carlos; OLIVERA ZALDÚA, María; LÓPEZ DEL RAMO, Joaquín; TORREGROSA, Juan Francisco; y PRIETO, Pablo R. (2011): "Nueva ecología de la información: el impacto digital en los diarios. Estudio preliminar". Documentación de las Ciencias de la Información, vol. 34, pp. 507-517. Madrid, Universidad Complutense de Madrid.

SÁNCHEZ VIGIL, Juan Miguel; MARCOS RECIO, Juan Carlos; y OLIVERA ZALDÚA, María (2011): "La nueva ecología de los documentos en la sociedad del conocimiento: aportaciones al proyecto infoscopos". En MARTÍNEZ COMECHE, Juan Antonio; MARCOS RECIO, Juan Carlos; y SÁNCHEZ VIGIL, Juan Miguel (eds.): Actas del VIII Seminario Hispano-Mexicano de Biblioteconomía y Documentación. Madrid, Universidad Complutense de Madrid, pp. 163-712. 